\title{
Stroke and Left Ventricular Assist Device (LVAD)
}

\author{
Robert P. From, David Hasan, Michael T. Froehler, Jennifer L. Goerbig-Campbell
}

Department of Anesthesia, Roy J. and Lucille A. Carver College of Medicine, Iowa City, USA.

Email: robert-from@uiowa.edu

Received October $6^{\text {th }}, 2012$; revised November $14^{\text {th }}, 2012$; accepted December $7^{\text {th }}, 2012$

\begin{abstract}
Treatment of ischemic stroke for a patient on left ventricular assist device (LVAD) by neurointerventional means is rare and many anesthesia providers are unfamiliar with both LVAD and neurointerventional protocols. Examples of this include: 1) filling for continuous-flow LVAD depend on preload and the flow is inversely related to afterload; as mean arterial pressure (MAP) increases above 80 to $90 \mathrm{~mm} \mathrm{Hg}$, flow decreases; 2) there may be no palpable pulse in patients with continuous flow LVADs; 3 ) pulse oximetry may not work when pump flow is high and native myocardial function is minimal; 4) increasing MAP above $80 \mathrm{~mm} \mathrm{Hg}$ potentially will maintain ischemic brain tissue - the penumbra - until flow is restored. This latter example creates a paradoxical management goal: increasing the mean arterial pressure (MAP) above $80 \mathrm{~mm} \mathrm{Hg}$ while maintaining ischemic brain tissue, may decrease flow to the LVAD. Finally, there is controversy regarding which type of anesthesia is most efficacious for neuro interventional procedures. We describe three patients on LVAD suffering ischemic stroke requiring anesthesia for embolectomy and angioplasty during neurointerventional radiology procedures.
\end{abstract}

Keywords: Stroke; Heart Failure; Endovascular; Embolectomy; Neurointerventional; HeartMate II ${ }^{\circledR}$; LVAD

\section{Introduction}

Anesthesia providers familiar with neurointerventional protocols are often unfamiliar with patients on left ventricular assist devices [(LVAD), these devices have a portable, self-contained, external pump which pushes blood from the left ventricle to the aorta] while noncardic anesthesiologists are unfamiliar with patients who require endovascular therapy for acute stroke. The limited time-critical period for endovascular therapy for stroke often requires care by anesthesia providers who are not expert in either field. General perioperative management principals of patients on LVAD have been discussed $[1,2]$. Specific reports for intra ab-dominal, laproscopic thoracotomy, and otologic procedures have also been described [3-6]. Care for patients with acute stroke on LVAD has not been discussed and presents a unique paradox.

Maintaining a mean arterial pressure (MAP) of at least $80 \mathrm{~mm} \mathrm{Hg}$ might be considered standard of care during most neurointerventional procedures involving stenotic lesions, but higher pressures are needed if angiographic evidence of ischemia is seen. On the other hand, flow through a LVAD is inversely related to afterload, or, in other words, MAP. As MAP increases above $90 \mathrm{~mm} \mathrm{Hg}$, flow decreases [7]. The relative infrequent occurrence of ischemic stroke and treatment by neurointerventional means, competition for blood pressure control combined with unfamiliarity of many anesthesia providers with LVAD, creates management challenges for care of these patients. We describe three patients on continuous flow LVAD (HeartMate II ${ }^{\circledR}$ LVAD, Thoratec Corporation, Pleasanton, CA) who developed thrombotic stroke treated by recanalization with angioplasty and stenting or mechanical thrombectomy. We discuss the anesthetic treatment challenges and review considerations for management in this select group of patients. Our Institutional Review Board determined that this project did not meet the regulatory definition of human subjects research and did not require review. Patients or family members of patients gave written consent for their case histories to be presented.

\subsection{Case 1}

A 35-year-old male with non-ischemic cardiomyopathy, continuous flow LVAD, and implantable cardiac defibrillator presented with anorexia, abdominal pain, bloody stools, and INR of 9.2. A "code stroke" was initiated at 6 $\mathrm{am}$, the morning following admission to the cardiovascular intensive care unit (CVICU) when marked left central facial paresis was noted. The patient had a calculated NIH Stroke Scale of $6(0=$ no stroke, $21-42=$ severe stroke). Multidetector computed tomography with an- 
giography (CTA) showed a distal occlusion of the middle cerebral artery consistent with an embolus. After arrival at the neurointerventional suite, his LVAD was attached to a battery console. Parameters included the following: pump flow $4.6 \mathrm{~L} / \mathrm{m}$; pump speed $9200 \mathrm{rpm}$; pulse index $5.4 \mathrm{~L} / \mathrm{min}$; and pump power 5.7 watts. No peripheral pulses were palpable. Initial blood pressure was $110 / 72$ $\mathrm{mm} \mathrm{Hg}$ using a blood pressure cuff with a mean of 90 $\mathrm{mm} \mathrm{Hg}$. The surgeon emphasized the importance of keeping the patient's blood pressure up with a MAP of at least $80 \mathrm{~mm} \mathrm{Hg}$; while the physician accompanying him from the CVICU cautioned that a MAP above $90 \mathrm{~mm} \mathrm{Hg}$ would decrease LVAD flow (which, in turn, would decrease cerebral perfusion rather than increase it). Prior to induction of general anesthesia, a right radial artery arterial line was placed with ultrasonic guidance. Ketamine was used for induction and maintenance continued with isoflurane. A phenylephrine infusion was used to maintain MAP between 80 and $90 \mathrm{~mm} \mathrm{Hg}$ throughout the procedure. Anesthesia start to stop time was 3 hours 17 minutes. Endovascular intervention consisted of recanalization of the right middle cerebral artery by balloon angioplasty and stenting. Occlusion was thought to be due to thromboembolization. The patient was extubated immediately following the procedure. At discharge, his neurologic symptoms had resolved.

\subsection{Case 2}

A 55-year-old female with non-ischemic cardiomyopathy and placement of continuous flow LVAD was admitted for colposcopy and biopsy. The procedure was completed with general endotracheal anesthesia. The next morning, the patient became acutely unresponsive, requiring urgent intubation. A basilar artery thrombus was identified, for which she underwent endovascular mechanical thrombectomy. Sedation and local anesthesia were provided by the neurointerventional service. Eleven days later, she developed right hemiparesis and aphasia due to a new left middle cerebral artery (MCA) embolism, and again underwent emergent thrombectomy. Monitored anesthesia care was provided. Seven days following this procedure, she suffered a third stroke characterized by acute left hemiparesis and unresponsiveness, requiring emergent intubation. She underwent a third thrombectomy procedure, this time for the right MCA. General anesthesia was provided. One month later, the patient was discharged to home care, was alert and oriented with dysarthria. The LVAD was connected to an external battery source during all her procedures. Blood pressure cuff was used during the first and last procedure; a radial artery catheter was placed with ultrasonic guidance for the second procedure. Anesthetic agents included etomidate $\left(\right.$ Amidate $\left.^{\mathbb{R}}\right)$, midazolam $\left(\right.$ Versed $\left.^{\mathbb{B}}\right)$, fentanyl, and isoflurane.

\subsection{Case 3}

A 61-year-old female with ischemic cardiomyopathy and continuous flow LVAD was admitted for pneumonia. Her hospital course was complicated by renal failure and femoral artery thrombosis and embolectomy. Five days following this procedure, she became drowsy and unresponsive. An embolus to the left MCA was identified. The patient underwent emergent clot aspiration of the left MCA. Monitoring and conscious sedation was performed by a nurse. Even though circulation was restored, her condition deteriorated with further evolution of the MCA stroke. She required intubation and blood pressure support. Her family declined further imaging studies and care was withdrawn.

\section{Discussion}

At our institution, 64 patients have received a HeartMate II ${ }^{\circledR}$ LVAD from May 2008 through May 2012. Nine patients developed strokes: four with ischemic stroke; one with hemorrhagic stroke; and four with embolic strokes one or more times.

Support for the failing heart includes medications and devices. Ventricular assist devices have evolved from large noisy consoles connected to patients by tubes to small implanted pumps with a driveline connected to a portable external power system. Devices can assist the right (RVAD) or left ventricle. When both ventricles are supported, the term biventricular assist device (BiVAD) is used. A RVAD pushes blood from the right ventricle to the pulmonary artery; a LVAD pushes blood from the left ventricle to the aorta [8].

The LVAD is used as a bridge for patients awaiting heart transplant or as destination therapy for patients unsuitable for transplantation. About $80 \%$ of LVAD implantations are for bridge therapy [9]. These devices can have a pumping chamber and valves which allow pulsatile flow or a rotary pump that creates continuous-flow, where no pulse is detectable unless the native heart is able to create enough cardiac output so that a pulse can be felt. For a LVAD, the inflow cannula is placed into the apex of the left ventricle and the outflow cannula is inserted into the base of the aorta above the coronary arteries. The HeartMate II $^{\circledR}$ is a continuous-flow LVAD Figure 1. It is the only pump approved by the FDA for bridge and destination therapy [10]. Flow through the device is determined by the speed of the pump and the differential pressure between the left ventricle and the aorta [10]. Increasing preload or decreasing afterload will increase flow through the LVAD.

Flow through the device is inversely related to afterload. Against low resistance (i.e. low afterload or MAP) the device can generate high flow, but as afterload increases there is insufficient power to overcome the resistance [11]. A MAP between 65 to $75 \mathrm{~mm} \mathrm{Hg}$ during 
LVAD support has been recommended [12]. Mean arterial pressures between 70 and $90 \mathrm{~mm} \mathrm{Hg}$ are typically used in clinical practice. When the HeartMate $\mathrm{II}^{\circledR}$ is connected to a Display Module, the display provides pump speed in revolutions per minute (rpm), estimated flow in $\mathrm{L} / \mathrm{min}$, power in Watts, and a pulsatility index (PI). Figure 2 A high PI may occur if the device is providing a low level of support or when the left ventricle is volume loaded; a low PI may occur when the device is providing high levels of support or the left ventricle is volume depleted. The PI typically ranges between 3 [13] and 6 .

Preload, or left ventricular filling, contributes to pump efficiency. Low volume status or high pulmonary artery pressure, which decreases filling of the right ventricle, will decrease pump output. Also, since the right ventricle $(\mathrm{RV})$ is not supported during LVAD support, unloading of the RV with an inotrope or pulmonary vasodilator, such as sildenafil $\left(\right.$ Revatio $^{\circledR}$ ), may be necessary. As the pump speed increases, the flow will be less pulsatile. Figure 3 shows a theoretical arterial wave form decrease as the speed increases from $4000 \mathrm{rpm}$ to $12,000 \mathrm{rpm}$. As the arterial wave form oscillations decrease, a perceptible pulse and oximetry become more difficult to obtain. Increasing preload with volume or inotropes or decreasing afterload with vasodilatation may be needed. Echocardiography can be helpful to define the function of the right ventricle, evaluating for thrombus, and in assessment of LVAD function itself.

If a thromboembolic arterial occlusion of cerebral vessels leaves a core area of neuronal death surrounded

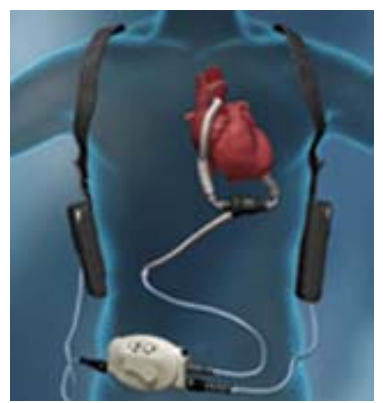

Figure 1. Internal and external components of the HeartMate $I I^{\circledR}$ left ventricular assist device. Image courtesy of the Thoratec Corporation.

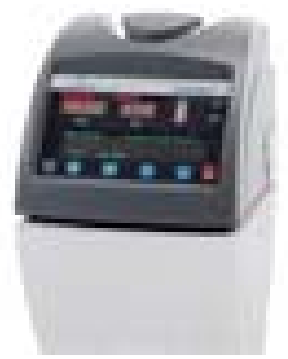

Figure 2. HeartMate $I^{\circledR}$ display module. Image courtesy of the Thoratec Corporation. by living but non-functioning tissue; this ischemic penumbra may be salvaged if perfusion is restored [14]. Four avenues of treatment have been proven efficacious. First, rapid "door-to-needle" intervention with intravenous tissue-type plasminogen activator (tPA) within up to 4.5 hours of stroke onset can dissolves the occlusion, although a target of $\leq 60$ minutes is the goal $[15,16]$. Second, endovascular therapy within six to eight hours following symptoms onset has been demonstrated to provide positive outcomes [17-19]. Third, direct intraarterial thrombolysis with prourokinase (ProUK) (not available in the US market) or tPA combined occasionally with mechanical thrombectomy up to 6 hours following onset of stoke symptoms $[17,20]$. Finally, transluminal angioplasty with or without stenting [21].

Perfusion to the penumbra by raising the blood pressure is controversial. Current American Heart Association guidelines state that drug-induced hypertension is not recommended for treatment of most patients with acute ischemic stroke [22]. However, small clinical studies suggest that drug induced hypertension could be used in select patients [22]. Treatment to lower blood pressure in acute ischemic stroke is not advocated unless other major end-organ damage is present $[14,22]$. Hypotension during induction of anesthesia may worsen the ischemic insult [23]. A Cochrane Review found that acute stroke is associated with high blood pressure in $75 \%$ of patients [24]; but there is little evidence to guide blood pressuremanagement in anesthetized or sedated patients [23].

Anesthesia for the three cases described consisted of three general anesthetics, one using monitored anesthesia care provided by an anesthesia team and two with nurse sedation. Conscious sedation during endovascular therapy for acute stroke is advocated by some [23,25-27] and cautioned by others [28-31]. A summary of arguments touting one method of anesthesia over another is presented in Table 1.

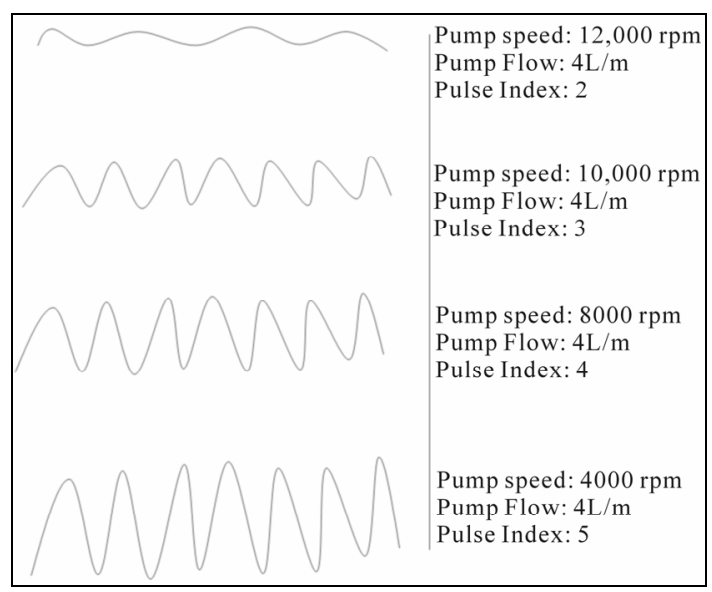

Figure 3. Theoretical arterial waveform at various Pump Speeds, Pump Flows and hypothetical Pulse Indexes of a left ventricular assist device (LVAD). 
Table 1. Arguments for using conscious sedation (CS) versus general anesthesia (GA) during endovascular procedures for acute stroke.

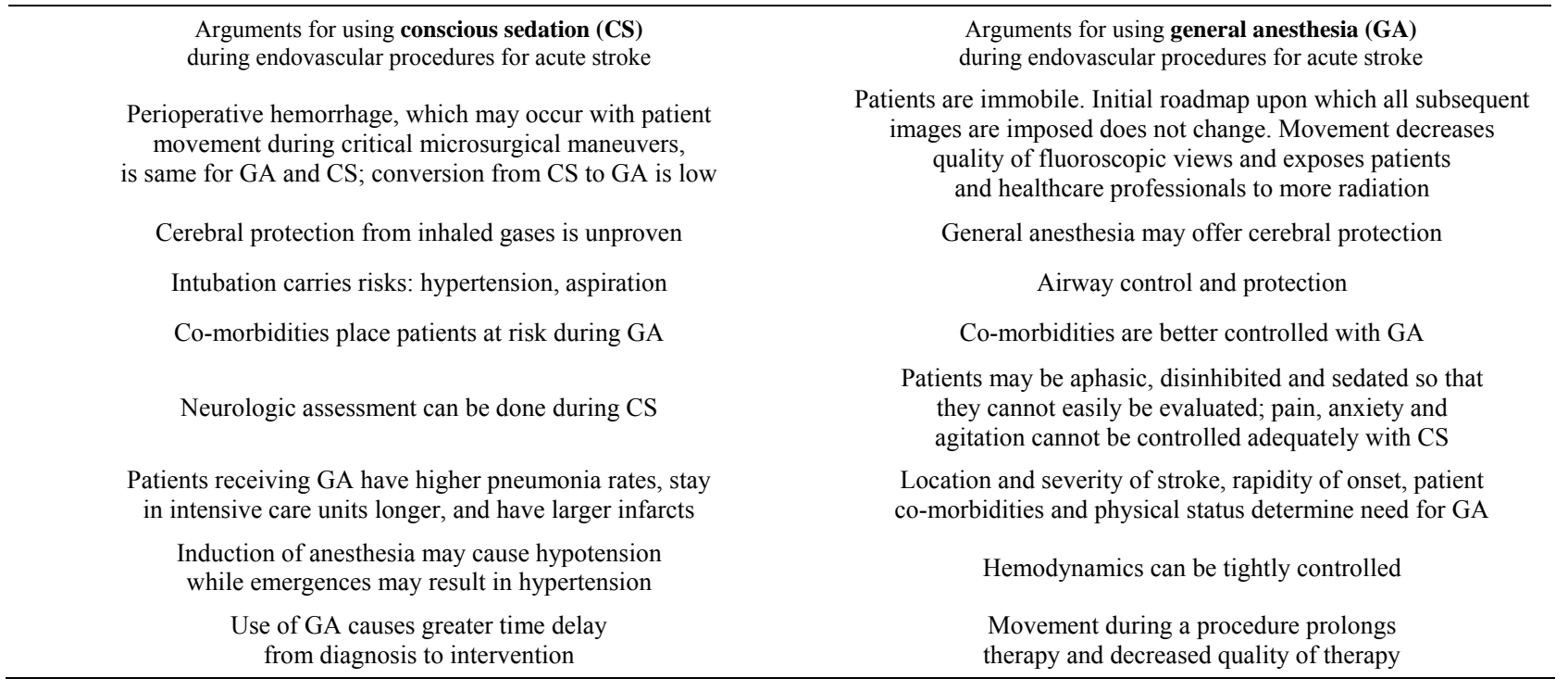

To settle the argument, we speculate that a large multicenter randomized control trial similar to one provided by the GALA Trial Collaborative Group (General anaesthesia versus local anaesthesia for carotid surgery (GALA): a multicenter randomized controlled trial) [31] will need to occur for endovascular procedures as it did for carotid surgery. The GALA trial concluded that the anesthesiologist and surgeon, in consultation with the patient, should decide which anesthetic to use on an individual basis.

\section{Conclusion}

Newer LVADs in combination with anticoagulation decrease the likelihood of ischemic stroke. Nevertheless, when stroke does occur, an endovascular intervention is needed; it is imperative that anesthesia care providers have an understanding of both LVAD and endovascular protocols to provide optimal care during a time-critical period.

\section{Acknowledgements}

The Authors wish to thank the patients and their families who graciously agreed to allow their case history to be presented in this report.

\section{REFERENCES}

[1] A. C. Nicolosi and P. S. Pagel, "Perioperative Considerations in the Patient with a Left Ventricular Assist Device," Anesthesiology, Vol. 98, No. 2, 2003, pp. 565-570. doi:10.1097/00000542-200302000-00038

[2] M. Oleyar, M. Stone and S. M. Neustein, "Perioperative Management of a Patient with a Nonpulsatile Left Ven-
tricular-Assist Device Presenting for Noncardiac Surgery," Journal of Cardiothoracic and Vascular Anesthesia, Vol. 24, No. 5, 2010, pp. 820-823.

doi:10.1053/i.jvca.2009.06.004

[3] A. L. Hoefnagel, R. Pasternak, A. E. Curle and M. P. Eaton, "Laparoscopic Gastric Bypass in a Patient with an Implanted Left Ventricular Assist Device," Journal of Cardiothoracic and Vascular Anesthesia, Vol. 26, No. 5, 2012, pp. 880-882. doi:10.1053/j.jvca.2011.02.012

[4] V. Kartha, W. Gomez, B. Wu and K. Tremper, "Laparoscopic Cholecystectomy in a Patient with an Implantable Left Ventricular Assist Device," British Journal of Anaesthesiology, Vol. 100, No. 5, 2008, pp. 652-655. doi:10.1093/bja/aen052

[5] M. E. Stone, W. Soong, M. Krol and D. L. Reich, "The Anesthetic Considerations in Patients with Ventricular Assist Devices Presenting for Noncardiac Surgery: A Review of Eight Cases," Anesthesia \& Analgesia, Vol. 95, No. 1, 2002, pp. 42-49. doi:10.1097/00000539-200207000-00007

[6] G. Samoukovic, M. Vassiliou, N. Giannetti, S. Al-Sabah, V. Lash and R. Cecere, "Laparoscopic Splenectomy in a Patient with a Heartmate ${ }^{\circledR}$ II Left Ventricular Assist Device," Journal of Laparoendoscopic \& Advanced Surgical Techniques, Part A, Vol. 21, No. 6, 2011, pp. 535-538. doi:10.1089/lap.2011.0169

[7] R. John, F. Kamdar, P. Eckman, et al., "Lessons Learned from Experience with over 100 Consecutive HeartMate II Left Ventricular Assist Devices," The Annals of Thoracic Surgery, Vol. 92, No. 5, 2011, pp. 1593-1599. doi:10.1016/j.athoracsur.2011.06.081

[8] R. Krishnamani, D. DeNofrio and M. A. Konstam, "Emerging Ventricular Assist Devices for Long-Term Cardiac Support," Nature Reviews Cardiology, Vol. 7, No. 2, 2010, pp. 71-76. doi:10.1038/nrcardio.2009.222

[9] M. C. Deng, L. B. Edwards, M. I. Hertz, et al., "Me- 
chanical Circulatory Support Device Database of the International Society for Heart and Lung Transplantation: Third Annual Report-2005," The Journal of Heart and Lung Transplantation: The Official Publication of the International Society for Heart Transplantation, Vol. 24, No. 9, 2005, pp. 1182-1187.

[10] K. B. Shah, D. G. Tang, R. H. Cooke, et al., "Implantable Mechanical Circulatory Support: Demystifying Patients with Ventricular Assist Devices and Artificial Hearts," Clinical Cardiology, Vol. 34, No. 3, 2011, pp. 147-152. doi:10.1002/clc. 20825

[11] O. H. Frazier, T. J. Myers, S. Westaby and I. D. Gregoric, "Clinical Experience with an Implantable, Intracardiac, Continuous Flow Circulatory Support Device: Physiologic Implications and their Relationship to Patient Selection," The Annals of Thoracic Surgery, Vol. 77, No. 1, 2004, pp. 133-142. doi:10.1016/S0003-4975(03)01321-3

[12] T. J. Myers, M. Bolmers, I. D. Gregoric, B. Kar and O. H. Frazier, "Assessment of Arterial Blood Pressure during Support with an Axial Flow Left Ventricular Assist Device," The Journal of Heart and Lung Transplantation: the Official Publication of the International Society for Heart Transplantation, Vol. 28, No. 5, 2009, pp. 423427.

[13] C. A. Thunberg, B. D. Gaitan, F. A. Arabia, D. J. Cole and A. M. Grigore, "Ventricular Assist Devices Today and Tomorrow," Journal Cardiothoracic and Vascular Anesthesia, Vol. 24, No. 4, 2010, pp. 656-680. doi:10.1053/j.jvca.2009.11.011

[14] M. J. Schneck, J. Biller and C. M. Loftus, "Interventions for Acute Ischemic Stroke," In: C. M. Loftus, Ed., Neurosurgical Emergencies, 2nd Edition, Thieme Medical Publishers, Inc., New York, 2008, pp. 102-114.

[15] G. C. Fonarow, E. E. Smith, J. L. Saver, et al., "Improving Door-to-Needle Times in Acute Ischemic Stroke: The Design and Rationale for the American Heart Association/American Stroke Association's Target: Stroke Initiative," Stroke, Vol. 42, No. 10, 2011, pp. 2983-2989. doi:10.1161/STROKEAHA.111.621342

[16] K. R. Lees, E. Bluhmki, R. von Kummer, et al., "Time to Treatment with Intravenous Alteplase and Outcome in Stroke: An Updated Pooled Analysis of ECASS, ATLANTIS, NINDS, and EPITHET Trials," Lancet, Vol. 375, No. 9727, 2010, pp. 1695-1703. doi:10.1016/S0140-6736(10)60491-6

[17] K. Becker, "Endovascular Treatment of Acute Stroke," Current Treatment Options in Neurology, Vol. 9, No. 6, 2007, pp. 463-469. doi:10.1007/s11940-007-0047-9

[18] W. S. Smith, G. Sung, J. Saver, et al., "Mechanical Thrombectomy for Acute Ischemic Stroke: Final Results of the Multi MERCI Trial," Stroke, Vol. 39, No. 4, 2008, pp. 1205-1212. doi:10.1161/STROKEAHA.107.497115

[19] Investigators Penumbra Pivotal Stroke Trial, "The Penumbra Pivotal Stroke Trial: Safety and Effectiveness of a New Generation of Mechanical Devices for Clot Removal in Intracranial Large Vessel Occlusive Disease," Stroke, Vol. 40, No. 8, 2009, pp. 2761-2768. doi:10.1161/STROKEAHA.108.544957

[20] A. Furlan, R. Higashida, L. Wechsler, et al., "Intra-Arte- rial Prourokinase for Acute Ischemic Stroke. The PROACT II Study: A Randomized Controlled Trial. Prolyse in Acute Cerebral Thromboembolism," JAMA: The Journal of the American Medical Association, Vol. 282, No. 21, 1999, pp. 2003-2011. doi:10.1001/jama.282.21.2003

[21] E. I. Levy, A. H. Siddiqui, A. Crumlish, et al., "First Food and Drug Administration-Approved Prospective Trial of Primary Intracranial Stenting for Acute Stroke: SARIS (Stent-Assisted Recanalization in Acute Ischemic Stroke)," Stroke, Vol. 40, No. 11, 2009, pp. 3552-3556. doi:10.1161/STROKEAHA.109.561274

[22] H. P. Adams Jr., G. del Zoppo, M. J. Alberts, et al., "Guidelines for the Early Management of Adults with Ischemic Stroke: A Guideline from the American Heart Association/American Stroke Association Stroke Council, Clinical Cardiology Council, Cardiovascular Radiology and Intervention Council, and the Atherosclerotic Peripheral Vascular Disease and Quality of Care Outcomes in Research Interdisciplinary Working Groups," Circulation, Vol. 115, No. 20, 2007, pp. 478-534. doi:10.1161/CIRCULATIONAHA.107.181486

[23] M. J. Davis, B. K. Menon, L. B. Baghirzada, et al., "Anesthetic Management and Outcome in Patients during Endovascular Therapy for Acute Stroke," Anesthesiology, Vol. 116, No. 2, 2012, pp. 396-405. doi:10.1097/ALN.0b013e318242a5d2

[24] C. Geeganage and P. M. Bath, "Interventions for Deliberately Altering Blood Pressure in Acute Stroke," Cochrane Database of Systematic Reviews, Vol. 3, 2008, Article ID: CD000039.

[25] A. Abou-Chebl, R. Lin, M. S. Hussain, et al., "Conscious Sedation versus General Anesthesia during Endovascular Therapy for Acute Anterior Circulation Stroke: Preliminary Results from a Retrospective, Multicenter Study," Stroke, Vol. 41, No. 6, 2010, pp. 1175-1179. doi:10.1161/STROKEAHA.109.574129

[26] R. Gupta, "Local Is Better than General Anesthesia during Endovascular Acute Stroke Interventions," Stroke, Vol. 41, No. 11, 2010, pp. 2718-2719. doi:10.1161/STROKEAHA.110.596015

[27] C. Nichols, J. Carrozzella, S. Yeatts, T. Tomsick, J. Broderick and P. Khatri, "Is Periprocedural Sedation during Acute Stroke Therapy Associated with Poorer Functional Outcomes?" Journal of Neurointerventional Surgery, Vol. 2, No. 1, 2010, pp. 67-70. doi:10.1136/jnis.2009.001768

[28] C. Brekenfeld, H. P. Mattle and G. Schroth, "General Is Better than Local Anesthesia during Endovascular Procedures," Stroke, Vol. 41, No. 11, 2010, pp. 2716-2717. doi:10.1161/STROKEAHA.110.594622

[29] L. B. Hemmer, C. Zeeni and D. K. Gupta, "Generalizations about General Anesthesia: The Unsubstantiated Condemnation of General Anesthesia for Patients Undergoing Intra-arterial Therapy for Anterior Circulation Stroke," Stroke, Vol. 41, No. 10, 2010, p. e573. doi:10.1161/STROKEAHA.110.588806

[30] C. A. Molina and M. H. Selim, "General or Local Anesthesia during Endovascular Procedures: Sailing Quiet in the Darkness or Fast under a Daylight Storm," Stroke, Vol. 41, No. 11, 2010, pp. 2720-2721. 
doi:10.1161/STROKEAHA.110.595447

[31] M. T. Froehler, J. T. Fifi, A. Majid, A. Bhatt, M. Ouyang and D. L. McDonagh, "Anesthesia for Endovascular Treatment of Acute Ischemic Stroke," Neurology, Vol. 79, No. 13, 2012, pp. 167-173. doi:10.1212/WNL.0b013e31826959c2
[32] GALA Trial Collaborative Group, S. C. Lewis, C. P. Warlow, et al., "General Anaesthesia versus Local Anaesthesia for Carotid Surgery," Lancet, Vol. 372, No. 9656, 2008, pp. 2132-2142.

doi:10.1016/S0140-6736(08)61699-2 